\title{
Central Retinal Microperimetry by Scanning Laser Ophthalmoscopy in Chiasmal Patients with Visual Inattention Temporally at Monocular Visual Acuity Testing
}

\author{
Hans C. Fledelius*, Mads Nis V. Andersen \\ Rigshospitalet, University Eye Department, Copenhagen, Demark \\ Email: ^hcfled@mail.dk
}

How to cite this paper: Fledelius, H.C. and Andersen, M.N.V. (2017) Central Retinal Microperimetry by Scanning Laser Ophthalmoscopy in Chiasmal Patients with Visual Inattention Temporally at Monocular Visual Acuity Testing. Open Journal of Ophthalmology, 7, 44-50.

https://doi.org/10.4236/ojoph.2017.71007

Received: December 14, 2016

Accepted: February 13, 2017

Published: February 16, 2017

Copyright $\odot 2017$ by authors and Scientific Research Publishing Inc. This work is licensed under the Creative Commons Attribution International License (CC BY 4.0).

http://creativecommons.org/licenses/by/4.0/

\begin{abstract}
Background: In a previous study, a specific visual behaviour was described in four chiasmal patients. It manifested as inattention/ignorance within the temporal visual space ("visual blocking") at monocular visual acuity testing. Moreover, in 3 out of the 4, the process of reading a text appeared cognitively impaired. Methods. As a supplement to conventional visual field testing, the present analysis focus was on microperimetry by Scanning Laser Ophthalmocopy (SLO). Our aim was to identify the perceptual retinal counterparts to the temporally located visual field defects as caused by the visual pathway lesion on a chiasmal level, possibly also to indicate the apparently ineffective saccadic movements underlying that only part of the line on the chart could be given. Results and Conclusions. The central retinal areas with lacking recognition by SLO testing were given by black rectangles expressing scotomatous points; they clustered in a zone extending nasally from the fixation area, as expected. The methodology further depicted an orderly fixation in two cases, and only minor deviation in two. Fixation saccades thus were considered by and large within normal and with only exceptional outlier points recorded. All considered, the shortcomings of the perceptual mechanisms underlying the lateralised visual inattention as observed in the four patients have remained without a satisfactory explanation.
\end{abstract}

\section{Keywords}

Microperimetry, Laser Ophthalmoscope, Monocular Visual Acuity Testing, Optic Chiasm

\section{Introduction}

At monocular visual acuity testing, some chiasmal patients with a temporal visual 
field defect can see only the optotypes nasally on the chart [1]. Though free to move their eyes from symbol to symbol, they cannot proceed along the line of letters, and the temporally located optotypes remain ignored, or "blocked". The inattention can be absolute (only marginal letters seen, to the nasal side on the chart) or with a graded blocking dependent on the visual acuity level actually tested for.

In the initial article on the issue [1], the main clinical data were given from the first four patients in our clinic in which the specific type of inattentive lateralising visual blocking behaviour was recognised. Analyses on a larger clinical scale were issued in subsequent articles [2] [3]. Apparently the saccades normally active for fixation and visual scanning in space were not triggered. It was hypothesised that this might be due to a lack of some visual input required for the automated visual search mechanisms, as usually assumed to be conducted on a supra-geniculate level.

With no obvious explanation for the specific visual behaviour, in a pilot study we performed microperimetry as achieved by Scanning Laser Ophthalmoscopy (SLO), a methodology otherwise primarily used for mapping central visual loss due to macular pathology. In our search for basic pathophysiological mechanisms, our object was to plot the retinal counterpart to the blocked visual pathway axonal transmission which, as assessed from the main clinical data, was due to lesions of the optic chiasm (pituitary adenoma in two, craniopharyngioma and skull trauma, one case each). Further, our aim was to evaluate the fixation saccades [4]-[9].

For comparison with the actual SLO recordings, our primary focus was on the Amsler chart findings, which described the scotomas' proximity to the fixation point, as subjectively outlined by the patient.

\section{Material and Methods}

With reference to the previous detailed clinical presentations [1] our first four patients with visual inattention temporally will here be given only by their key findings. The initial lesions had been diagnosed 2 - 19 years prior to the SLO microperimetries, with current neuroophthalmological updates also related to the microperimetries under study. Informed consent was given to the SLO examinations and to publishing the data.

Patient No 1, a 53-year-old female with a regular follow-up since a brain concussion in 1980. Since then: visual acuity testing revealed a permanent absolute temporal blocking behaviour of each eye, however with a full line 1.0 acuity given on the chart when binocular. Visual field testing revealed only minute bitemporal paracentral scotomas. Reading a standard text was heavily disturbed, also when using both eyes, and remained so for more than a decade.

Patient No 2, a 46-year-old female with unilateral visual acuity loss in her moderately amblyopic eye (due to anisomyopia) after surgery for a craniopharyngioma. Further, she had bitemporal visual field defects and a markedly disturbed reading.

Patient No 3, a 58-year-old male bricklayer with insidious left eye visual loss. 
After surgery for pituitary adenoma the left eye increased in acuity from 0.16 to 0.6 , however with graded temporal blocking. He forwarded no reading complaints, now or earlier, but also claimed "never to have been a reader".

Patient No 4, a 42-year-old male for whom reading normal text (papers, books) had become impossible despite a visual acuity of 0.6 in both eyes. Soon after surgery for pituitary adenoma he could read a fine test smoothly (N4 1/4), and his pre-surgery bitemporal relative visual field defects had normalised, as also the temporally lateralised visual inattention at initial monocular testing of both eyes.

Monocular visual acuity was determined subjectively, with best glasses in a trial frame and using 1) mirror reading of $6 \mathrm{~m}$ distance projector optotypes, mainly with four symbols shown in each frame, and 2) free space testing on a conventional Snellen chart at 3 metres.

Regarding reading disability we primarily weighted the actual complaints, e.g. "previously smooth reading, now almost impossible", as in the three academics out of the four patients under study. Subsequent binocular testing was done using a standard reading text (authorised by the Danish Ophthalmological Society, 1966). With add for near work the disabled patients typically recognised small print single monotypes (N4 1/4), but gave up virtual reading if words were longer than 5 - 7 letters.

A full ophthalmic evaluation was performed including visual field examination by tangent screen, kinetic (Goldmann) and static computerized (Octopus) perimetry specifying the temporally located visual defects as apparent in 7 out of the 8 eyes under study. The defects were relative only (i.e. more extensive for the small Goldmann object (I, 4e) than for the larger standard object of (IV, 4) and without definite macular splitting. Further, we compared the SLO microperimetry findings to the patients' subjective marking on an Amsler chart, which evaluated the central 10 degrees around the fixation point, under a reading distance of $35 \mathrm{~cm} \mathrm{[9]} \mathrm{[10].}$

In SLO-microperimetry computer-guided laser stimuli $(633 \mathrm{~nm})$ are presented to the central retina during low-level infrared illumination for fundus visualisation. When arousing computer-guided visual stimulation in a 10 degree test-zone around the fovea the patient responds by pressing a button, as is also done in static and kinetic perimetry. The investigation can thus monitor the subject's visual behaviour-perceptual losses and fixation strategies-by viewing the test stimuli on the continuous real-time image of the central fundus [5] [6] [7] [11].

As previously shown in normal subjects and in patients with retinal lesions of the macula in our clinic, test results obtained by SLO microperimetry were comparable to those obtained by computer-based macular programmes (e.g. Octopus M1) [11].

\section{Results}

Figure 1 presents the microperimetric ocular right and left eye fundus correlates 

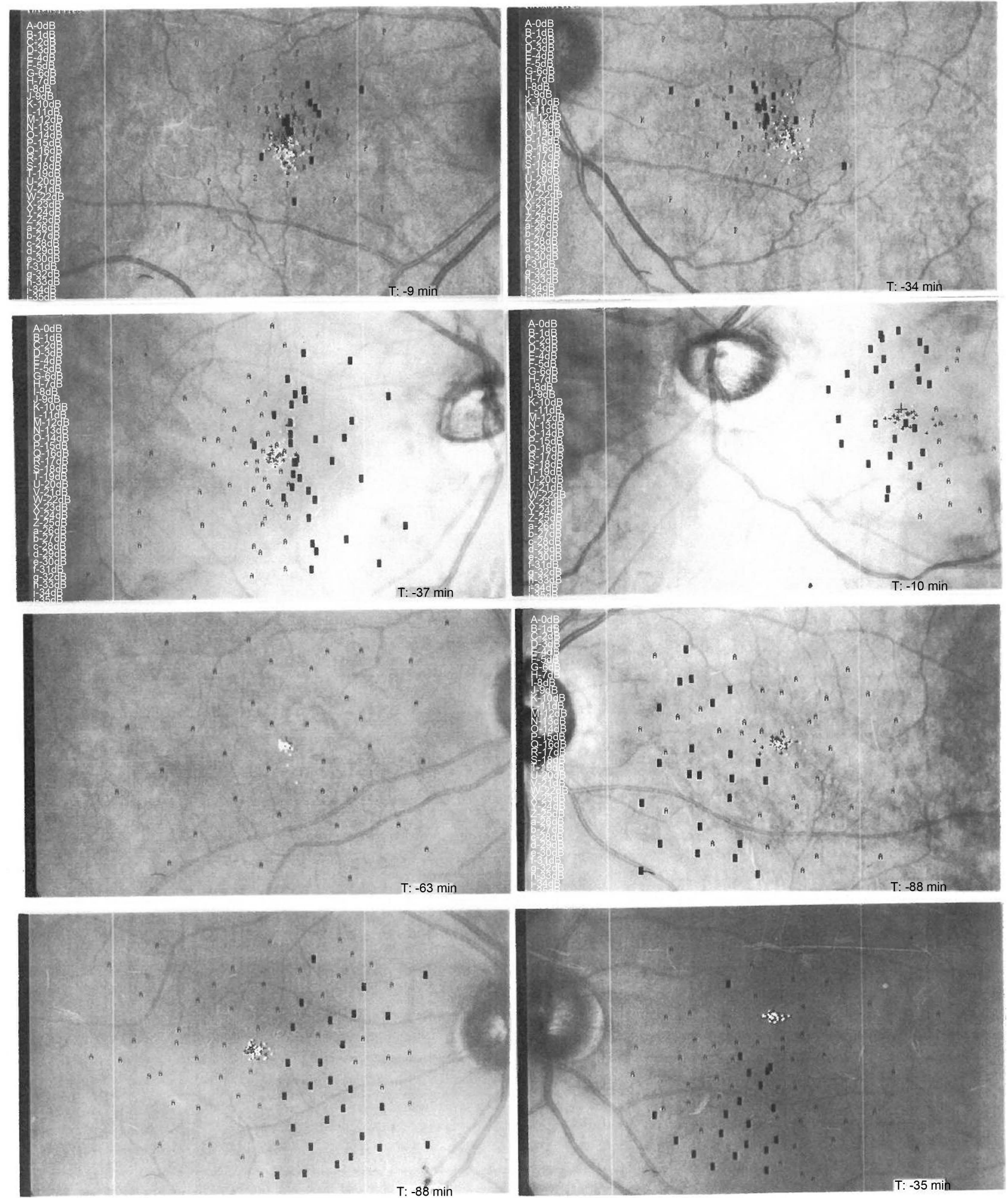

Figure 1. The SLO findings by microperimetry in right and left eyes, in patients 1 (at top) through 4 (at bottom). The minute white dots mark the fixation zone, the dark rectangles the retinal points where stimuli are not perceived.

to the central visual field drop-outs. The fine white cluster of dots marks the fixation area of the subject, the minute black dots the laser stimuli that were recognized within the testing zone, and the black rectangles the retinal scotoma 
correlates, i.e. where stimuli were applied but not perceived. The scotoma points being located mainly nasally for the fovea corresponded to the central part of the temporal visual field drop-outs in the 7 eyes affected.

\section{Discussion}

In clinical praxis, visual field defects usually are compared to intracranial pathology, as demonstrated by CT and MRI imaging, however without including retinal correlates. Multifocal ERG can give functional clues regarding topical involvement of the retina, but has, to our knowledge, not been used in the present context. The method was not established in our clinic when our pilot study investigating SLO microperimetry findings was performed, in the search for possible central and parafoveal retinal correlates of the visual field scotomas as demonstrated in chiasmal patients with a lateralising inattentive monocular visual chart performance ("blocking temporally"). The question was: would the microperimetric findings merely reflect the visual field dropouts suggesting a point-to-point representation? Or, might deficient visual recognition in space be combined with some degree of additionally curtailed transmission, possibly leading to inadequate cortical processing and abnormal search by visual scanning saccades during conventional visual acuity testing? We should add here that usually the SLO microperimetry technique is used for evaluation of topical retinal function in association with diseases of the macula, and it cannot replace conventional visual field examinations.

Regarding fixation pattern, the white dots by SLO microperimetry (Figure 1) generally depicted a quite steady fixation, as also asked for by the examiner according to the testing algorithm. The "monocular" patient No. 3 and the "binocular" patient No. 4 were the most precise and showed the smallest fixation areas, with only a minimum of searching eye movement. Patient No. 2 had kinked optic nerves with inferior conus, and a temporally downwards slanting of the horizontal fundus raphe was suggested from the cluster of white fixation marks. The SLO evaluation of patient No. 1 was first performed 15 years after her head trauma, when she was still visually stigmatised. Out of the four patients, she had the largest fixation area, probably to illustrate a tentative saccadic compensation for her fixation trouble. The monocular temporal blocking behaviour of each eye had remained permanent, but even with a smooth binocular compensation during visual acuity testing (full Snellen lines given using both eyes together) her reading of a text, a book for instance, was still impaired. All the way through, this functional deficit had been quite out of proportion to the minute paracentral bitemporal scotomas as demonstrated by the conventional visual field techniques, including the Amsler chart [1].

The scotoma correlates on the retina as given by the SLO technique are mainly seen in the papillomacular region (Figure 1 ), which means within a $5^{\circ}-8^{\circ}$ zone nasally for what strictly represents the foveal fixation. However, in patient No. 1 , who exposed the widest fixation search areas, a few "temporal" scotoma outlier points were also observed. The same held for patient No. 2 whose subjectively 
recorded left eye Amsler chart scotoma paradoxically transgressed the fixation zone. Theoretically this might be due to a moderate anisomyopia-related amblyopia since childhood. In patients No. 3 and 4 the SLO scotoma indications strictly held the nasal retinal location that corresponded to the visual field defects, and there was no indication of "temporal" overlap.

As a practical conclusion we found a good agreement with the customary perimetry procedures. This is not embarrassing, since the recording of the laser SLO stimulus is based on conscious recognition on a supra-geniculate processing level. The SLO microperimetry thus presented no new keys for explaining the factual visual apraxia in the pilot sample under study.

All considered, in the four patients we found no evidence that visual information was lost for the brain in addition to what was stated already by conventional visual field testing. The retinal stimuli actually recorded appeared determined by the degree of axon-related block of the anterior visual pathway transmission as due to the para-chiasmal pathology, and supplementary neuro-psychological evaluations were without specific clues [3]. So far we have no clear concept regarding why some such patients visually compensate smoothly, whereas others with apparently similar lesions seem to have a marked cognitive difficulty associated with for instance reading, or with other everyday visual demands in space.

\section{References}

[1] Fledelius, H.C. (2004) Chiasmal Pathology Causing Inability to Access Information in the Temporal Visual Field. A Report of a Specific Visual Behaviour Manifest on Visual Acuity Testing. Neuro-Ophthalmology, 28, 77-85. https://doi.org/10.1076/noph.28.2.77.23740

[2] Fledelius, H.C. (2009) Temporal Visual Field Defects Are Associated with Monocular Inattention in Chiasmal Pathology. Acta Ophthalmologica, 87, 769-775.

[3] Fledelius, H.C. (2016) Cognitive Visual Disturbances in Chiasmal Disorders. A Review on Exceptional Visual Disabilities as Based on 30 Years' Clinical Experience. Journal of Medical \& Surgical Pathology, 1, 117.

[4] Timberlake, G.T., Mainster, M.A., Peli, E., Augliere, R.A., Essock, E.A. and Lawrence, E.A. (1986) Reading with a Macular Scotoma. 1. Retinal Location of Scotoma and Fixation Area. Investigative Ophthalmology \& Visual Science, 27, 1137-1147.

[5] Van der Velde and Jalkh. (1992) Microperimetry with the Scanning Laser Ophthalmoscope: A New Technique for Correlating Retinal Sensitivity with Anatomy. Chibret International Journal of Ophthalmology, 9, 36-48.

[6] Culham, L.E., Fitzke, F.W., Timbwel, K.W. and Marshall, J. (1993) Assessment of Fixation Stability in Normal Subjects and Patients Using a Scanning Laser Ophthalmoscope. Clinical Vision Science, 6, 551-561.

[7] Møller, F., Sjølie, A.K. and Bek, T. (1996) Quantitative Assessment of Fixational Eye Movements by Scanning Laser Ophthalmoscopy. Acta Ophthalmologica Scandinavica, 74, 578-583. https://doi.org/10.1111/j.1600-0420.1996.tb00739.x

[8] Møller, F. and Bek, T. (1998) The Relation between Visual Acuity and the Size of fixational Eye Movements in Patients with Diabetic and Non-Diabetic Macular Disease. Acta Ophthalmologica Scandinavica, 76, 38-42. https://doi.org/10.1034/j.1600-0420.1998.760107.x 
[9] Amsler, M. (1953) Earliest Symptoms of Diseases of the Macula. British Journal of Ophthalmology, 37, 521-537. https://doi.org/10.1136/bjo.37.9.521

[10] Kanski, J.J. (2003) Amsler Grid, in Clinical Ophthalmology. 5th Edition, Butterworth Heinemann, Edinburg, 392-393.

[11] Andersen, M.V.N. (1996) Scanning Laser Ophthalmoscope Microperimetry Compared with Octopus Perimetry in Normal Subjects. Acta Ophthalmologica Scandinavica, 74, 135-139. https://doi.org/10.1111/j.1600-0420.1996.tb00057.x

Submit or recommend next manuscript to SCIRP and we will provide best service for you:

Accepting pre-submission inquiries through Email, Facebook, LinkedIn, Twitter, etc. A wide selection of journals (inclusive of 9 subjects, more than 200 journals)

Providing 24-hour high-quality service

User-friendly online submission system

Fair and swift peer-review system

Efficient typesetting and proofreading procedure

Display of the result of downloads and visits, as well as the number of cited articles Maximum dissemination of your research work

Submit your manuscript at: http://papersubmission.scirp.org/

Or contact ojoph@scirp.org 